\title{
VIOLÊNCIA INTRAFAMILIAR: CAMINHOS PARA O ENFRENTAMENTO NA SAÚDE PÚBLICA
}

\author{
FAMILY VIOLENCE: PATHWAYS FOR COPING IN PUBLIC HEALTH
}

\section{Adriana Carvalho dos Santos \\ Especialista em Saúde da Família pela Casa de Saúde Santa Marcelina.}

E-mail: adrianna.csantos@gmail. com

\section{Mara Lisiane de Moraes dos} Santos

Doutora em Ciências da Saúde, Universidade Federal de Mato Grosso do Sul.

E-mail: maralisi@globo.com

\section{Débora Dupas G. Nascimento}

Doutora em Ciências, Universidade de São Paulo. Pesquisadora em Saúde Pública, Fiocruz Mato Grosso do Sul.

E-mail: ddupas@uol.com.br

\section{Resumo}

A violência é um assunto relevante na área da saúde, pois suas consequências causam grande impacto na integridade do indivíduo, afetando seu bem-estar físico, moral, espiritual e mental. A violência intrafamiliar, por sua vez, representa um importante desafio aos profissionais da saúde pública, que se sentem inseguros e despreparados para manejar essa situação. Frente a isso, o presente estudo se propôs a identificar como os serviços de saúde abordam as situações de violência intrafamiliar no cotidiano da prática. Tratase de uma pesquisa do tipo bibliográfica, realizada nos últimos cinco anos. Foram consultadas duas bases de dados, Lilacs e Scielo, e utilizados os descritores "violência" e "serviços de saúde". Os 12 artigos que compuseram a amostra atenderam aos critérios de inclusão previamente estabelecidos. Constatou-se que a maioria dos autores optou pelo delineamento qualitativo e escolheu a entrevista para realizar a coleta de dados. Grande parte dos estudos foi desenvolvida na região Sudeste do País, tendo a amostra composta por profissionais de saúde, usuários e profissionais de outras áreas. Observou-se que a violência intrafamiliar é comumente relacionada à violência contra a mulher e a perspectiva diagnóstica do problema é amplamente discutida, apesar da escassez de relatos de experiência. Nota-se que os profissionais de saúde se sentem pouco habilitados para lidar 
com a questão e têm dificuldade para reconhecê-la como um problema de interesse epidemiológico e social, o que reflete no baixo número de notificações e impede a visualização dos efeitos desse fenômeno sobre os indivíduos, famílias e comunidades.

Palavras-chave: Violência; Violência Doméstica; Serviços de Saúde.

Abstract: Violence is an important issue in health care because it takes toward deep impacts on the personal integrity, affecting physical, moral, spiritual and mental well-being. Family Violence, in turn, represents an important challenge for public healthcare professionals who are insecure and unprepared to manage this situation. Faced to it, this study was performed to identify how health services deal with these issues on its routine. It is a data literature survey performed in the past five years. Two databases, Lilacs and Scielo were surveyed and the keywords "violence" and "health services" were researched. The sampled 12 articles matched the inclusion criteria previously established. We found that most authors used a qualitative design and choose interview procedures as data collection. Most of the studies were developed in the Southeast of the country and the sample data was composed by healthcare professionals, patients and professionals from other areas. We observed that domestic violence is commonly related to violence against women and the diagnostic perspective of this problem is widely discussed, despite the lack of experience reports. During the study we noticed that healthcare professionals feel themselves poorly qualified to deal with this problem and does not recognize it as a trouble for social and epidemiological interest. It reflects the low number of violence cases notifications and blocks the view of the effects of this phenomenon on individuals, families and communities.
Keywords: Violence; Domestic Violence; Healthcare Services.

\section{Introdução}

A violência pode ser definida como a ação humana capaz de provocar a morte de outros homens ou impactar em seu bemestar físico, moral, espiritual ou mental. ${ }^{1}$ Esse fenômeno é relevante e desperta o interesse da área da saúde face ao efeito que suas consequências têm na integridade do indivíduo e do coletivo. ${ }^{2}$

Um exemplo de violência que se configura como um grande desafio para gestores e profissionais do Sistema Único de Saúde (SUS), é a violência intrafamiliar, que "caracteriza-se pelas variadas formas de violência interpessoal: agressão física, abuso sexual, abuso psicológico, negligência, abandono, maus-tratos, entre outras". ${ }^{3: 15}$ A violência doméstica pode ser praticada por um ou mais autores, que mantenham com as vítimas laços familiares, matrimoniais, algum grau de parentesco ou vínculo afetivo, muitas vezes ligado as relações de poder. ${ }^{3}$

A conjunção dos setores e saberes presentes no campo da saúde pode contribuir significativamente para a redução da violência. Para que esse objetivo seja alcançado, Dahlberg \& Krug $^{4}$ alertam para a adoção de quatro etapas essenciais: 1) analisar o fenômeno da violência identificando sua amplitude, características e consequências; 2) pesquisar as causas e os fatores associados ao problema, verificando riscos e possibilidades de intervenção; 3) utilizar os dados para elaborar ações de prevenção, efetuando seu planejamento, execução, monitoramento e avaliação; 4) disseminar as iniciativas que pareçam promissoras, divulgando seus custos e resultados.

Conhecer a visão que o trabalhador da saúde tem sobre a violência também 
é imprescindível, uma vez que suas convicções tendem a influenciar na forma de ofertarem assistência e cuidado. ${ }^{5} \mathrm{Em}$ estudo desenvolvido com profissionais da saúde mexicanos, foram relacionadas diferentes concepções acerca da violência contra as mulheres e foi possível verificar que alguns profissionais preferem ignorar a existência do problema, enquanto outros sentem compaixão pelas vítimas e as motivam a sair dessa situação. Existem, ainda, aqueles que concebem a violência como um atraso cultural e educacional e tecem críticas severas às vítimas e os que condenam fortemente a violência contra a mulher, considerando-a um delito e uma violação dos direitos humanos. ${ }^{6}$

Segundo Moreira ${ }^{7}$, idosos, mulheres, crianças e adolescentes constituem os principais alvos de agressões que, em sua maioria, ocorrem nos domicílios e apresentam elevado nível de reincidência.

De acordo com o sistema de Vigilância de Violência e Acidentes (VIVA) ${ }^{8}$ a violência sexual representa o principal motivo de atendimento a crianças e adolescentes nos serviços de referência, superando as agressões físicas. Entre crianças de zero a nove anos, a violência sexual correspondeu a $44 \%$ dos atendimentos, enquanto a violência física gerou $29 \%$ dos atendimentos a essa faixa etária. ${ }^{3}$

Em relação ao gênero feminino, dados do Mapa da Violência de 2012 revelam que o Brasil ficou em 7ạ colocação no quesito "número de homicídio de mulheres", diante dos demais países pesquisados, que representavam um total de $84 .^{9}$

Apesar dos números expressivos, Herrera \& Agoff ${ }^{6}$ enfatizam que algumas pessoas desistem de buscar auxílio por se sentirem duplamente violentadas pelo abusador e também pelo profissional de saúde. As autoras evidenciaram que os pacientes nem sempre são inqueridos sobre violência doméstica, mesmo diante de indícios e sinais que podem caracterizar a ocorrência de algum tipo de violência.

Dossi ${ }^{10}$ constatou que muitas vítimas que recorreram aos serviços de saúde, ao sofrer uma agressão, relataram não receber diagnóstico relacionado à violência e notaram o desinteresse do profissional, mesmo quando lhes comunicavam a situação. Esse estudo ressalta a insegurança dos profissionais de saúde, que não recebem capacitação adequada e periódica que possibilite desenvolver habilidades para melhor manejo dos casos e para a realização da notificação.

Com a publicação da Portaria Ministerial 104, de 25 de janeiro de $2011^{11}$, as violências doméstica e sexual, entre outras, foram incluídas na lista de doenças, agravos e eventos de notificação compulsória, ${ }^{7}$ sendo esta

... obrigatória a todos os
profissionais de saúde médicos,
enfermeiros, odontólogos,
médicos veterinários, biólogos,
biomédicos, farmacêuticos e
outros no exercício da profissão,
bem como os responsáveis por
organizações e estabelecimentos
públicos e particulares de saúde e
de ensino, em conformidade com
os arts. 70 e 8 o, da Lei $n^{\circ}$ - 6.259 , de
30 de outubro de $1975 .{ }^{11}$

Deslandes $^{12}$ explica que a resistência dos profissionais para assumir uma notificação de maus tratos fundamentase em aspectos como: a) experiências desfavoráveis (retaliações sofridas ou relatadas); b) receio de que a vítima seja institucionalizada; c) compreensão de que se trata de um problema exclusivamente familiar; d) insegurança quanto ao diagnóstico, medo de cometer um engano; e) concepção de que $o$ atendimento prestado deve se limitar aos cuidados das lesões; f) desconfiança sobre suas reais condições de intervenção.

A mesma autora salienta a necessidade das instituições oferecerem maior suporte 
aos profissionais de saúde:

Seporumladoéderesponsabilidade profissional tal notificação (ética e legalmente), por outro é a instituição que deve garantir esta conduta profissional, protegendo o indivíduo de "arcar" com todas as pressões, compartilhando a responsabilidade pelo caso. Há que se criar uma "rotina institucional" para a notificação, identificandose etapas e atribuições: um "quem faz o quê" entre os diversos atores, médicos (as) e enfermeiros (as), chefes de equipe médica e de enfermagem, chefias da emergência, serviço social da emergência, Direção do hospital e Secretaria Municipal de Saúde. ${ }^{12: 91}$

No contexto da saúde pública, o levantamento das informações relativas à violência intrafamiliar são de extrema relevância para: 1) verificar quais são os tipos de violência e acidentes, com consequente identificação do perfil epidemiológico; 2) fazer o levantamento dos fatores de risco existentes; 3 ) criar estratégias para a promoção da saúde e para a prevenção dos agravos; 4) desenvolver políticas públicas para o enfrentamento dessa problemática, articulando diferentes setores da sociedade e 5) contribuir para a melhoria das ações de assistência, recuperação e reabilitação. ${ }^{13}$

Considerando a magnitude do tema violência para o setor da Saúde e os desafios que diariamente são impostos aos profissionais da área, faz-se necessário investigar os caminhos já percorridos para o enfrentamento do problema.

Nesse sentido, o presente estudo se propôs a identificar, por meio da literatura brasileira, como os serviços de saúde abordam as situações de violência intrafamiliar no cotidiano da prática. Estima-se que as informações levantadas possam contribuir para o debate e para o desenvolvimento de novas ações e estratégias em torno dessa questão.
Método

Trata-se de uma revisão bibliográfica que, segundo Cervo \& Bervian, ${ }^{14: 65-66}$ é aquela em que se "procura explicar um problema a partir de referências teóricas publicadas em documentos", a fim de analisar as colaborações científicas ou culturais já existentes sobre um assunto ou problema específicos.

Neste estudo, pretendeu-se fazer o levantamento de artigos que discutissem acerca da violência no espaço doméstico e suas interfaces com os serviços de saúde. Para isso, foram selecionadas duas bases de dados: Lilacs (Literatura Latino-Americana e do Caribe em Ciências da Saúde ${ }^{15}$ e Scielo (Scientific Electronic Library Online). ${ }^{16}$

A base de dados Lilacs $^{15}$ é o mais amplo e relevante índice de literatura científica da América Latina e do Caribe. Já a base Scielo ${ }^{16}$, corresponde a uma biblioteca eletrônica que contém uma extensa seleção de periódicos científicos nacionais.

Para efetuar a busca nessas bases, foram utilizados os descritores "violência" e "serviços de saúde", ambos cadastrados no DeCS (Descritores em Ciências da Saúde) até o final do ano de 2013. Com o descritor "violência", houve um resultado de 2039 artigos na base Scielo ${ }^{16}$ e 6522 na base Lilacs $^{15}$. Quando foram combinados os descritores "violência" e "serviços de saúde", foram encontrados 22 artigos na Scielo ${ }^{16}$ e 86 na Lilacs. ${ }^{15}$

O total de artigos levantados foi submetido ao crivo de critérios de inclusão e exclusão, previamente estabelecidos. Foram incluídos os estudos publicados a partir do ano de 2009, em língua portuguesa, cujo texto estava disponível integralmente na internet e que retratavam aspectos da realidade brasileira. Teses, dissertações, cartilhas e estudos que não abordaram a temática, não foram considerados. 


\section{Artigo de Original}

Após a leitura integral dos artigos selecionados, verificou-se que apenas 12 correspondiam aos critérios estabelecidos no método, sendo que sete foram localizados somente na Scielo ${ }^{16}$, três somente na Lilacs ${ }^{15}$ e dois em ambas as bases.

Para este estudo, optou-se por um recorte dos anos de 2009 a 2013, procurando levantar dados atuais acerca do assunto em questão. Foram encontradas publicações em todos os anos desse período, sendo duas publicações em 2009, duas em 2010, quatro em 2011, duas em 2012 e duas em 2013.

\section{Resultados e Discussão}

O quadro 1 apresenta a relação de artigos que compuseram a amostra deste estudo.

\begin{tabular}{|c|c|c|c|}
\hline ARTIGO & AUTORES & $\begin{array}{l}\text { DELINEAMENTO } \\
\text { METODOLÓGICO }\end{array}$ & LOCAL DE ESTUDO \\
\hline A1 & VILLELA, Wilza Vieira et al. & Qualitativo & São Paulo \\
\hline $\mathrm{A} 2$ & $\begin{array}{c}\text { VIEIRA, Elisabeth Meloni; PERDONA, } \\
\text { Gleici da Silva Castro; SANTOS, Manoel } \\
\text { Antonio dos. }\end{array}$ & Quantitativo & São Paulo \\
\hline A3 & VIEIRA, Luiza Jane Eyre de Souza et al. & Quantitativo & Ceará \\
\hline A4 & HESLER, Lilian Zielke et al. & Qualitativo & Rio Grande do Sul \\
\hline A5 & SAURET, Gerard Viader et al. & Qualitativo & Pernambuco \\
\hline A6 & SCHRAIBER, Lilia Blima et al. & Quantitativo & São Paulo \\
\hline A7 & PEDROSA, Claudia Mara. & Qualitativo & São Paulo \\
\hline A8 & GRANJA, Edna; MEDRADO, Benedito. & Qualitativo & Pernambuco \\
\hline A9 & $\begin{array}{l}\text { CAVALCANTI, Maria de Lourdes Tavares; } \\
\text { SOUZA, Edinilsa Ramos de. }\end{array}$ & Qualitativo & Rio de Janeiro \\
\hline A10 & LIMA, Maria Luiza Carvalho de et al. & Quanti-Quali & $\begin{array}{c}\text { Rio de Janeiro / } \\
\text { Pernambuco / Paraná } \\
\text { /Amazonas / Distrito } \\
\text { Federal }\end{array}$ \\
\hline A11 & $\begin{array}{c}\text { BERGER, Sônia Maria Dantas; GIFFIN, } \\
\text { Karen Mary. }\end{array}$ & Qualitativo & Rio de Janeiro \\
\hline A12 & $\begin{array}{l}\text { SILVA, Aline Gaudard e; MORAES, Claudia } \\
\text { Leite; REICHENHEIM, Michael Eduardo. }\end{array}$ & Quantitativo & Rio de Janeiro \\
\hline
\end{tabular}

Quadro 1. Estudos inseridos na revisão de literatura acerca as violências no espaço doméstico e suas interfaces com os serviços de saúde. 
Foi possível constatar que a maioria dos autores optou pelo delineamento qualitativo, o que correspondeu a sete artigos; $;^{17,18,19,20,21,22,23}$ quatro adotaram o delineamento quantitativo ${ }^{24,25,26,27}$; e em um artigo se observou a associação dos dois métodos. ${ }^{28}$ No que se refere à estratégia para coleta dos dados, nove utilizaram entrevista ${ }^{17,18,19,20,28,22,25,26,13}$; quatro aplicaram questionários ${ }^{28,24,25,26}$; quatro recorreram a registros de observação ${ }^{17,19,22,23}$; um consultou fichas de notificação ${ }^{27}$; e dois consideraram informações obtidas durante oficinas de intervenção. ${ }^{17,21}$

Quanto à região em que se desenvolveu o estudo, verificou-se a seguinte disposição: oito no Sudeste; ${ }^{17,18,28,21,24,25,26,23}$ quatro no Nordeste; ${ }^{19,28,22,27}$; dois no Sul; ;0,28 um no Norte ${ }^{28}$; e um no Centro-Oeste. ${ }^{28}$

No que tange à amostra, cinco estudos foram realizados apenas com profissionais de saúde; ${ }^{17,18,20,28,22}$ cinco apenas com usuários de serviços de saúde ${ }^{21,24,25,13,27}$, sendo que, em um deles, os dados foram obtidos por meio de fichas de notificação; e duas amostras incluíram profissionais de saúde e de outras áreas, tais como segurança pública e social. ${ }^{19,23}$

Dos 12 artigos, sete trazem como assunto central a violência contra o gênero feminino. Somente dois ${ }^{19,24}$ tiveram o gênero masculino como principal objeto de estudo, mesmo assim, trazendo, entre outros assuntos, discussões a respeito da violência contra a mulher. Os demais discutem a violência contra crianças e adolescentes ${ }^{22}$ e a violência contra idosos. ${ }^{18,28}$ A maior produção científica voltada para violência contra o gênero feminino pode estar associada ao fato de a mulher ser a principal vítima da violência doméstica e sexual em todas as fases da vida, como apontam informações registradas no sistema VIVA. ${ }^{8}$

Quanto aos objetivos dos estudos, foi possível categorizá-los da seguinte forma: quatro $^{18,19,28,23}$ avaliaram $\mathrm{O}$ atendimento prestado às vítimas; quatro $24,25,26,27$ traçaram uma análise epidemiológica acerca de fenômenos associados à violência; dois ${ }^{20,22}$ investigaram a representação social da violência na perspectiva do profissional de saúde; um ${ }^{21}$ apresentou resultados de uma intervenção; e, por fim, um ${ }^{17}$ investigou a representação social da violência na visão do profissional de saúde, além de relatar uma intervenção realizada.

Os estudos demonstraram que a presença de determinados fatores favorecem a ocorrência da violência, como uso de álcool e drogas, condição socioeconômica desfavorável (como morar de aluguel ou favor) e a presença de histórico de violência familiar tanto na vida do ofensor quanto da vítima. ${ }^{24,25,26,27}$

Nota-se a similaridade entre os dados obtidos neste estudo com os achados de Marinheiro et $\mathrm{al}^{29}$ que observaram que o uso de drogas pelo parceiro íntimo, a condição socioeconômica desfavorável e o histórico de violência familiar são produtores de violência psicológica. Já os riscos para violência física envolvem o uso de drogas pelo parceiro, baixa escolaridade e histórico de violência familiar. No que diz respeito à violência sexual, destacam que a condição socioeconômica e a história de violência familiar favorecem a sua ocorrência, enquanto que o uso de drogas pelo parceiro, a condição socioeconômica e a história de violência familiar representam riscos para todos os tipos de violência.

Vieira et $\mathrm{al}^{26}$ ressaltam que a escolaridade é um importante fator de proteção para as mulheres. Os autores verificaram que $48,2 \%$ das vítimas de sexo feminino tinham até oito anos de escolaridade, $43,4 \%$ tinham entre nove e 11 anos de estudo e somente $8,2 \%$ havia frequentado a escola por mais de 12 anos. Tais dados coincidem com os achados de Marinheiro et $\mathrm{al}^{29}$, que constataram que menos anos de escolaridade correspondem a maior índice de violência. Em sua amostra, $32,4 \%$ das mulheres que sofreram violência 
física tinham cursado até o primeiro grau, enquanto as agressões a mulheres mais instruídas corresponderam a $19,8 \%$.

Ficou evidenciada a recorrência de discussões referentes à insuficiência estrutural dos equipamentos de saúde para o atendimento às vítimas, assim como a escassez de ambientes adequados para o acolhimento das queixas e de salas que preservem a privacidade daqueles que buscam ajuda e orientação. A necessidade de estruturação da rede e o conhecimento e a implantação dos fluxos para encaminhamento dos casos foram outros pontos frágeis identificados. ${ }^{17,18,19,28,23}$

Cavalcanti \& Souza, ${ }^{18}$ ao tratar da violência contra idosos, notam que a assistência enfrenta grandes limitações como a superlotação dos hospitais, que recebem nas emergências pacientes que deveriam ser acolhidos na Atenção Básica, escassez de leitos de retaguarda e falta de uma rede estruturada para o adequado suporte social.

Notificar casos de violência aparece como mais uma fragilidade, como destacam Lima et $\mathrm{a}^{28}$, ao realizarem uma análise da assistência oferecida à população idosa nos serviços hospitalares. Os autores constataram que os hospitais não vêm seguindo a orientação de notificar os casos suspeitos e confirmados de violência, pois grande parte dos serviços que participaram do estudo não realizava notificação sistemática dos casos. A subnotificação também foi discutida no estudo de Kind et $\mathrm{al}^{30}$ no qual os profissionais entrevistados identificaram como obstáculos à notificação questões como medo de retaliação, constrangimento frente às situações de violência contra a mulher, dificuldade em preencher a ficha, entre outros.

Alguns autores também ressaltam a falta de capacitação dos profissionais para tratar do tema violência, a ausência de uma escuta qualificada para identificar fatores de risco e possíveis vítimas, a insegurança na abordagem do tema, além da dificuldade para lidar com a diversidade de grupos sociais atingidos pelo problema (mulheres, idosos, crianças, adolescentes etc.). ${ }^{17,18,19,28,23}$

Foi possível perceber que em determinados casos os profissionais que prestam atendimento às vítimas acabam por responsabilizá-las pela violência sofrida. No estudo de Villela et al ${ }^{23}$ verificou-se que nas situações em que a mulher é vítima de violência sexual, mas se descobre que o fato se deu após o uso de substância psicoativa, é comum que profissionais, tanto da saúde quanto da segurança pública, a responsabilizem pelo ocorrido. Esse julgamento também foi identificado no estudo de Kiss \& Schraiber, ${ }^{31}$ em que grande parte dos profissionais entrevistados considera a mulher responsável pela situação que vivencia, sendo esta o resultado de suas escolhas.

Nos artigos em que são relatadas experiências de intervenção, fica claro o interesse e a opção dos autores por estratégias que propiciam maior nível de reflexão, sensibilização e qualificação da escuta do profissional para trabalhar com as situações de violência encontradas em sua prática diária. ${ }^{17,21}$

\section{Considerações Finais}

Neste estudo, verificou-se que a violência intrafamiliar é comumente relacionada à violência praticada contra o gênero feminino. Nota-se, contudo, que outros grupos também são afetados, tais como idosos, crianças e adolescentes. 0 homem, por vezes, aparece como o principal ofensor, porém, são escassos os estudos que se propõem a investigar o lugar do gênero masculino nesse contexto, seja no papel de vítima, seja no papel de ofensor.

Discute-se amplamente a perspectiva diagnóstica do problema da violência, 
porém há poucos registros de intervenções que possam direcionar as ações dos profissionais de saúde em seu cotidiano. Observa-se que esse assunto mobiliza sentimentos de angústia, insegurança e revolta nos profissionais, que se consideram pouco hábeis para lidar com o problema. Nota-se também uma naturalização e um ocultamento do fenômeno, que é compreendido como algo de caráter particular e de difícil manejo.

A falta de capacitação específica, a inadequada estruturação da rede de apoio e a dificuldade em exercer uma atuação multidisciplinar efetiva dificultam a reflexão e o reconhecimento, por parte dos profissionais, de que a violência doméstica é um problema de interesse epidemiológico e social, que ultrapassa o caráter particular e invade a esfera da saúde pública. Consequentemente, essa concepção mais restrita acerca do problema reflete no baixo número de notificações e impede a visualização da amplitude e do impacto que esse fenômeno causa nos indivíduos, nas famílias e nas comunidades.

\section{Referências}

1. Minayo MCS, Souza E R. Violência e saúde como um campo interdisciplinar e de ação coletiva. Hist. Cienc. Saúde -Manguinhos. 1997 Nov; 4(3): 513-531.

2. Minayo MCS. A inclusão da violência na agenda da saúde: trajetória histórica. Ciênc. Saúde Coletiva. 2007; 11(Sup): 1259-1267.

3. Ministério da Saúde (BR). Painel de Indicadores do SUS. Brasília: MS; 2008.

4. Dahlberg LL, Krug EG.. Violência: um problema global de saúde pública. Ciênc. saúde coletiva. 2007; 11(Sup) 1163-1178.

5. Souza MKB, Santana JSS. Concepções de enfermeiros gestores municipais de saúde sobre violência. Revista Enfermagem (UERJ). 2007 Jan-Mar; 15(1): 94-9.

${ }^{6}$. Herrera C, Agoff C. Dilemas del profissional médico ante la violencia de pareja em México. Cad. Saúde Pública. 2006 Nov; 22(11): 2349-2357.

7. Moreira, TNF. A construção do cuidado: o atendimento às situações de violência doméstica por equipes de saúde da família [dissertação]. São Paulo: Universidade de São Paulo, Faculdade de Saúde Pública; 2012.

8. Ministério da Saúde (BR). Vigilância de Violências e Acidentes. Brasília: MS; 2007.

9. Waiselfisz JJ. Mapa da Violência 2012. Caderno complementar 1: homicídio de mulheres no Brasil. Instituto Sangari; 2012.

10. Dossi AP. Violência doméstica: o que se espera do profissional de saúde? [dissertação de mestrado]. Araçatuba: Faculdade de Odontologia; 2006.

${ }^{11}$. Ministério da Saúde (BR). Portaria no. 104, de 25 de janeiro de 2011. Define as terminologias adotadas em legislação nacional, conforme o disposto no Regulamento Sanitário Internacional 2005 (RSI 2005), a relação de doenças, agravos e eventos em saúde pública de notificação 


\section{Artigo de Original}

compulsória em todo o território nacional e estabelece fluxo, critérios, responsabilidades e atribuições aos profissionais e serviços de saúde. Brasília: Diário Oficial da União; 2011.

${ }^{12}$. Deslandes SF. O atendimento às vítimas de violência na emergência: "prevenção numa hora dessas?". Ciênc. saúde coletiva; 1999; 4(1): 81-94.

13. Secretaria municipal da saúde (SP). Sistema de informação para a vigilância de violências e acidentes - SIVVA - Manual de Preenchimento Ficha de Notificação de Casos Suspeitos ou Confirmados Secretaria. São Paulo: SMS; 2007.

14. Cervo AL, Bervian PA. Metodologia científica. 5.ed. São Paulo: Prentice Hall; 2002.

15. Literatura Latino-Americana e do Caribe em Ciências da Saúde [homepage na internet]. Acesso em 08 de dezembro 2013. Disponível em: http: http://lilacs.bvsalud.org.

16. Scientific Electronic Library Online [homepage na internet]. Acesso em 08 de dezembro 2013. Disponível em: http: http://www.scielo.org.

17. Berger SMD, Giffin KM. Serviços de saúde e a violência na gravidez: perspectivas e práticas de profissionais e equipes de saúde em um hospital público no Rio de Janeiro. Interface (Botucatu); 2011 Abr-Jun; 15(37): 391-405.

18. Cavalcanti MLT, Souza ER de. Percepções de gestores e profissionais de saúde sobre a atenção aos idosos vítimas de violências no município do Rio de Janeiro (RJ, Brasil). Ciênc. saúde coletiva. 2010 Set; 15(6): 2699-2708.

19. Granja E, Medrado B. Homens, violência de gênero e atenção integral em saúde. Psicol. Soc. 2009 Jan-Abr; 21(1): 25-34.

20. Hesler LZ, Costa MC da, Resta D G, Colomé I C S. Violência contra as mulheres na perspectiva dos agentes comunitários de saúde. Rev Gaúcha de Enferm. 2013 Mar; 34 (1): 180-186.

${ }^{21}$. Pedrosa CM. A construção de uma ferramenta social para promoção da saúde e dos direitos das mulheres. Paidéia (Ribeirão Preto). 2009 Jan-Abr; 19(42): 123-129.

${ }^{22}$. Sauret GV, Carneiro RM, Valongueiro S, Vasconcelos MGL de. Representações de profissionais da saúde sobre famílias de crianças e adolescentes vítimas de violência. Rev. Bras. Saude Mater. Infant.; 2011 Jul-Set; 11(3): 265-273.

23. Villela WV, Vianna LAC, Lima LFP, Sala DCP, Vieira TF, Vieira M L, et al. Ambiguidades e contradições no atendimento de mulheres que sofrem violência. Saude Soc. 2011 Jan-Mar; 20(1): p.113-123.

24. Schraiber LB, Barros CRS, Couto MT, Figueiredo WS, Albuquerque FP de. Homens, masculinidade e violência: estudo em serviços de atenção primária à saúde. Rev. Bras. Epidemiol. 2012 Dez; 15(4): 790-803.

${ }^{25}$. Silva AG, Moraes $\mathrm{CL}$, Reichenheim ME. Violência física entre parceiros íntimos: um obstáculo ao início do acompanhamento da criança em unidades básicas de saúde do Rio de Janeiro, Brasil?. Cad. Saúde Pública. 2012 Jan-Jul; 28(7): 1359-1370.

26. Vieira EM, Perdona GSC, Santos MA dos. Fatores associados à violência física por parceiro íntimo em usuárias de serviços de saúde. Rev. Saúde Pública. 2011 Ago; 45(4): 730-7. 


\section{Artigo de Original}

27. Vieira LJES, Ferreira RC, Moreira GAR, Gondim APS, Araújo MAL, Silva RM da. Fatores associados a sobreposição de tipos de violência contra a mulher notificada em serviços sentinela. Rev. Latino-Am. Enfermagem; 2013 Jul-Ago; 21(4): [08 telas].

28. Lima MLC de, Souza ER de, Acioli RML, Bezerra ED. Análise dos serviços hospitalares clínicos aos idosos vítimas de acidentes e violências. Ciênc. saúde coletiva. 2010 Set; 15 (6): 26872697.

29. Marinheiro ALV, Vieira EM, Souza L de. Prevalência da violência contra a mulher usuária de serviço de saúde. Rev. Saúde Pública. 2006 Ago; 40 (4): 604-10.

30. Kind L, Orsini MLP, Nepomuceno V, Gonçalves L, Souza G A de, Ferreira M F F. Subnotificação e (in)visibilidade da violência contra mulheres na atenção primária à saúde. Cad. Saúde Pública. 2013 Set; 29(9): 1805-1815.

31. Kiss LB, Schraiber LB. Temas médico-sociais e a intervenção em saúde: a violência contra mulheres no discurso dos profissionais. Ciênc. saúde coletiva. 2011 Mar; 16(3): 1943-1952. 